\title{
The Lived Experience of Internationally Recruited Nurses in Oman a Phenomenological Study
}

Glenn Ford D. Valdez PhD, RN ( $\square$ glennfordvaldez@yahoo.com )

Oman College of Health Sciences - Dhofar https://orcid.org/0000-0002-2799-8216

Arcalyd Rose R. Cayaban MSN, RN, RM

Sultan Qaboos University https://orcid.org/0000-0001-8665-9372

Muna B. Ajzoon PhD, RN

Oman College of Health Sciences -Dhofar

Gina M. Molina PhD, RN

Oman College of Health Sciences - North Batinah

Visitacion O. Bumalay

Oman College of Health Sciences - Dhakliya

\section{Research Article}

Keywords: Internationally recruited nurses, Lived experience, Acculturation, Migrant nurse, Oman nurses

Posted Date: March 13th, 2021

DOl: https://doi.org/10.21203/rs.3.rs-322435/v1

License: (c) (1) This work is licensed under a Creative Commons Attribution 4.0 International License.

Read Full License 


\section{Abstract}

Aim- The study aimed at exploring the lived experience of internationally recruited nurses in Oman that seeks to know how culture affects their adjustment process. The study also explored the acculturation that nurses undertake to adapt to a new culture from a personal and nursing perspective and the study also identified the support mechanisms that are in place to assist in the transition.

Methods- The study is a qualitative phenomenological study defined as an approach to understanding people's everyday life experience utilizing Collaizi's 9 Phenomenological Steps. The samples were taken from four (4) major governorates in Oman namely Dhofar, Dhakliya, North Batinah \& Muscat. Out of 45 Nurses, 18 met the inclusion criteria and volunteered to undergo the structured phone interviews and conferences. Data Analysis was performed using the analytical software NVivo ver 12.

Results-18 nurses consented to participate in the interview, most Internationally recruited nurses holding a bachelor's degree with an average age of 33.5 years. Most participants reported challenges in their transition to Oman upon being deployed. The primary area of concern arose from not having a structured ethical recruitment system and orientation programs.

Conclusion- There is a need for a more systematic and ethical recruitment strategy that enhances IRN's full integration into the host country's social, religious, and cultural system. It was also found that language is an integral part of the IRN's assimilation therefore the need for a formal and structured training and orientation program must be in place in both recruiting agencies and the catchment facilities in Oman

\section{Introduction}

The Sultanate of Oman is one of the largest Arabic gulf countries and one of the founding members of the Gulf Cooperation Council (GCC). It lies within the borders of Saudi Arabia and the United Arab Emirates in the west and Yemen in the south. Muscat is the largest city and the capital (Index Mundi: Oman Demographic Profile 2014). Professional nursing in Oman has grown rapidly since the country's healthcare modernization started in the 1970's. Nursing has been seen as the fastest-growing profession in the Sultanate over the past ten years (Al Maqbali \& Al Omari et al.. (2019). Oman has struggled with its rapidly growing population, the rapid expansion of healthcare services and the severe shortages of well-trained local nurses to meet the demands (Hill \& Chen, 1996).In recent years the global demands for nurses have increased dramatically and the recruitment of internationally trained nurses in western countries and in the Gulf states has continually grown over the years. In Oman based on the Ministry of Health statistics in 2017 on human resource for health there are approximately 19,938 practicing nurses in the Ministry of Health, Private sector \& Government Non-MOH Institutions in the country $48 \%$ of which are Omanis and $52 \%$ were internationally trained nurses (IRN's) $(\mathrm{MOH}, 2017)$. The continuous effort for a widespread Omanization in all sectors has lessened the gaps in the number of IRN's and local Nurses although efforts have made good progress in the ratio of nurses locally trained and IRN's the gap 
requirements are still being filled by experienced nurses coming from India, The Philippines, and Sri Lanka (Minority Nurse: Professional Nursing in Oman, 2013). The Middle East, Oman in particular, is a unique setting with an intertwined mix of culture, beliefs, and religion that is a crucial factor that needs to be sought when working in such a given milieu. The nurse of any foreign origin must comply with the mores, norms, values, and belief in this region. In a broader sense foreign-trained nurses who migrate encounter new culture, undergoes a process often called acculturation. This is a process in which new groups of newcomers make sense of the surroundings and acquire new knowledge to enable them to produce the conduct allowed among the established members of the community to recognize them as competent (Bond \& Bond, 1994). The literature has shown that low cultural sensitivity in nursing care leads to dissatisfaction with that care also leads also to poor quality of health outcomes for patients and families in contrast to cultural considerate care which is more capable of providing competent care (Chenowethm, et.al 2006, Cooper, et.al 2007). A couple of studies have touched on the concept of lived experience among IRN's the settings were in the perspective of the UK environment which created insight on the cultural adaptation of IRN's in an English environment (Matiti \& Taylor, 2005) (Patel-Kerai, Harcourt, Rumsey, \& Naqvi, 2010) (Daniel, Chamberlain, \& Gordon, 2001). A similar quantitative study on the cultural adaptability Registered nurses in the GCC identified that language, work ethics, religious practices and climatic differences were significant adjustments that the Filipino nurses in the GCC need to be adapted to. (Valdez, 2016) (Al-Yateem \& Al -Yateem, 2014). The study endeavors to explore the cultural lived experiences of the IRN's in Oman. The results of the study may be utilized in the future to further strengthen the acculturation programs given to newly hired IRN's that would help them immerse smoothly into the system, therefore, providing the much sought culturally competent care for the local population.

\section{Aims and research questions}

The study is a first of its kind in the Sultanate of Oman, the country's unique cultural environment is sought to have great influence in the quality-of-care IRN's are providing their patients. This research may further enhance the future in improving the induction and transition training for newly recruited IRN's. A contextualize influence of Omani culture and the adaptation mechanism utilized by the IRN's can be an additional knowledge in making future decisions in hiring, retention as well as nurse's professional development.

This study is aimed to understand the core experience of IRN's and how their cultural background affects their adjustment process. To specifically investigate the acculturation, process the nurses undertake to adapt to a new culture from a personal and nursing perspective and to identify the support mechanisms that are in place to assist in the transition.

The study seeks to explore the IRN's lived experiences and answer the following research questions. How does IRN's cultural background affect their adjustment process? What acculturation process does IRN's utilize to adapt to the new cultural environment? What support mechanism is available for the IRN's? 


\section{Methods}

\section{Study design}

In this qualitative phenomenological study, nurses were interviewed in detail about their cultural lived experience in Oman. Phenomenology can be defined as an approach to understanding people's everyday life experiences (Polit \& Tatano Beck, 2014). This phenomenological study tried to understand the subjective experiences of the participants by looking back at their lived experiences being an internationally recruited nurse in Oman. Bracketing of the participants' experiences was done without bias and prejudice (Sandelowski., Docherty, S \& Emden,1997) .The study adopted the phenomenological approach by Colaizzi utilizing the nine phenomenological steps (Colaizzi, 1978).

\section{Characteristics of the participants}

Nurses in Oman can be categorized based on the institutions they are working with; the majority of the Nurses population are employed in the Ministry of Health. Omanization in the Ministry of Health is at $62 \%$, other Non- $\mathrm{MOH}$ - government facilities $34 \%$ and $4 \%$ in the private sector $(\mathrm{MOH}, 2017)$ this significantly implies that the gaps are still being filled by IRN's. In the Ministry of Health alone $48 \%$ $(9,556)$ are IRN's. Many Nurses work full time in government healthcare facilities, hospitals, clinics, and health centers (Nurse Minority, 2013). Foreign nurses are primarily hired from India, the Philippines, SriLanka, Pakistan, African Countries and few from neighboring Arab countries such as Jordan \& Egypt. The participants in this present study were 18 nurses from four (4) major governorates in Oman namely Dhofar, Dhakliya, North Batinah \& Muscat working under the Ministry of Health Oman healthcare institutions. These study participants comprised IRN (Internationally recruited nurses) that have recently joined Oman Nursing service not longer than 36 months. Initial purposive sampling was conducted based on the list of participants recommended by the respective regional nursing directorates.

\section{Data Collection}

\section{Training background and preparation}

Researchers were faculty academicians and academic heads of nursing colleges in Oman with varying experiences in the clinical field. Researchers took qualitative research training and courses from their graduate schools. In addition, most have participated in qualitative studies prior to this study. Therefore, they are deemed qualified and capable of understanding the participant's cultural lived experience.

The participants chose where to interview or where they are most comfortable giving the interview. The selection of the interview venue is essential to allow the participants to freely share their insights and discussion about the topic and it also enhances the credibility of data gathered (Matiti \& Taylor, 2005). The original data gathering was set to be conducted using face to face or in-person interviews but due to the rising cases of COVID-19 by March 2020 and stricter health protocols the data collection procedure was adjusted to limit or avoid face-to-face contact. By March 2020 over the phone interviews were initiated, the study participants were contacted and informed about the best time for them to receive the 
interview. Once dates were scheduled the researchers started the interview. In the interview sessions through phone calls wherein audio calls are recorded with the consent and permission of the participants. A typical interview will last from 40 minutes to 120 minutes. The researchers conducted a detailed interview using a semi-structured checklist to avoid differences or mistakes in the data collection process. Live experience consists of the average daily experience of an individual in the background of normal pursuits and emphasis on what is real and true to that of an individual (Fain, 2004). The following questions were asked. Which country do you come from? How long have you been here? How were you recruited to come to work in Oman? Can you describe what preparation you had for moving to Oman? Tell me your story about your experiences? How was your first week's experience in Oman? Has it changed? , In what way? Can you please describe your experiences at work? , Can you describe any aspect of your cultural background that has influenced your adaptation personally and professionally? Are there any suggestions that your recruitment agency could have made to improve your transition from one country's culture to another? Data gathering and analysis were done concurrently. Each and after the interview, the analyst listened to the documented data, reread the transcript, and coded the data. Newly gathered data are pre-analyzed and compared for likenesses and variances. The transcribed data were autonomously coded by the five authors, and results were deliberated to reach complete agreement among study authors.

\section{Data analysis}

Verbatim transcription of audio recordings was done by the researchers and reviewed and verified by the interviewers. NVivo ver. 12 (QSR International Victoria, Australia) qualitative data analysis software was used to store, manage and order the data. The transcribed and clustered data were examined using the system defined by Collaizi. In the initial stage of analysis, the researcher read the transcribed data numerous times, aiming at the context of data and the participant's responses to screening out significant cues. Related phrases and expressions were grouped and organized according to extracted statements. This is followed by the extraction of themes by grouping similar contents and statements. Saturation was reached when there are no emerging or different statements appeared in the succeeding interviews and repeated themes appeared in the course of repeated interviews.

\section{Quality of data and data management}

Quality is established by following Sandelowskis et.al (1997) method following four standards. The first is to maintain credibility by starting with open-ended questions to permit participants to freely express their thoughts and experiences. Emotions, thoughts, and experiences of the researchers were excluded in the bracketing and are screened repeatedly through analysis of the interviews. Credibility is enhanced by reconfirming statements that are abstract to the participants by scheduling a second interview. Second suitability is proven based on the depth of data collection until saturation is met. The researchers extracted ideas from abstracted statements by reverting back to the participants to explain in detail their thoughts. Third, the auditability confirms that there is no contradiction and a sound conclusion can be induced. This study received feedback from co-authors on the results of the study. Interpretation and 
analysis are consulted with the team to ensure that interpretations are seen in different perspectives due to differences in clinical and academic backgrounds of the researchers, this later on aids in identifying preconceived ideas about the statements. The fourth conformability is maintained and obtained without influence from the researchers. Separation of preconceptions, assumptions, and notions about the study was recorded to narrow in on the experiences of the participants. Finally, three study participants reviewed the summary of results if it captures the essence of their experience.

\section{Ethical Considerations}

Ethical approval was obtained from the Institutional Review Board of the Center for Scientific Research Ministry of Health in the Sultanate of Oman (Approval No.MOH/CSR/19/10678). Anonymity and confidentiality among the participants were maintained in the course of the data collection process. The participants were informed about the non-bearing nature of the research data collection process and were informed that they can withdraw at any stage of the process voluntarily. Prior to data collection, and informed consent was read with the participant seeking their consent and permission to proceed with the recorded telephone interview. Transcription was done by coding the participant to maintain the respondent's anonymity.

\section{Results}

The quantitative data profiling of the IRN's were presented in tabulated form (Table 1) and the qualitative data was organized and classified into nine key themes.

- Reasons to work as a nurse in Oman

- Hiring and recruitment experience

- Preparation for transition

- Barriers and challenges

- New work environment experience

- Variations in practice

- Adjustment and acculturation

- Improving the hiring process

- Impermanence and Omanization

\section{Profile of the participating IRN's}

The demographic details obtained regarding the profile of the IRN's (Table 1) shows that the majority of them are in the Philippines and India, females with an average age of 33.5 years, with Bachelor's degree in nursing are widely working in the wards and general settings as a general nurse or junior staff nurse with an average of 24-36 months experience within Oman and at least 6- 10 years' experience outside Oman. 


\section{Reasons to work as a nurse in Oman}

This study reveals the various reasons IRN's choose to work in Oman. These reasons can be broadly clustered into both personal and professional reasons. Personal reasons have stemmed from economic reasons that aim to further better the opportunities of earning more than what is regularly being received in their countries of origin and to help family members back home with their finances. There is also a desire to move in order to join relatives or family members who are already in the Sultanate. Other personal reasons comprise the desire to immerse in a new cultural environment and to relocate in a country wherein practice in terms of religion, belief, and values are tolerated.

R2 "I decided to work in Oman to help my family financially, it's difficult for nurses in the Philippines to find a stable high-paying job. Oman is a safe country, knowing this after talking to my friends who have worked here, I felt relieved because they told me that Oman is a good county"( pg.4. Line 136-139)

$R 11$ "one of the reasons for going out from the Philippines and working in Oman is that nurses in the Philippines are under-compensated" (pg.20. Line 773).

$\boldsymbol{R} \mathbf{7}^{\prime \prime}$ I previously worked in Saudi Arabia, a man who worked in Oman before explained to me that people of a different religion can practice safely and freely their beliefs, I am a Christian and that is very important for me especially if I encounter a difficult situation " (pg.14 line 483-48)

Professional reasons arise from the IRN's desire to learn new skills, broaden their clinical experience, have access to more advanced equipment and to enhance professional development through in-service education with some saying they left to have better work situations.

$R \mathbf{R}$ "One of my cousins and an uncle worked in Oman, they recommended me to an agency and encouraged me to work here, and they described Oman as almost the same with India in terms of climate and people. Oman has warm weather and friendly people and that there is no discrimination or racism in this country. I can also work on my specialization which is the intensive care unit and I was given a good salary offer" (pg.19. Line 690-695)

R10 "In the Philippines during that time I think I enjoyed waiting because of the opportunity of being promoted. I think the reason why I finally resigned is because of the stress I'm having at work. Feel I am under-compensated with the workload I was having then. I don't have time to enjoy my social life. Even at home, I was still working. I was even being called during my off days. I was also stressed with a bunch of paperwork. I started to feel the burnout from work. (pg.21 line 794-799)

\section{Hiring and recruitment experience}

The majority of the IRN's were recruited through agencies in their countries of origin. Mediating agencies advertise the required staff and their specialization based on the vacancies provided by the hiring institutions in Oman. The hiring process will take about 6 months to 2 years. The applicants are required to meet the expected minimum requirements such as passing the Oman Prometric exam, minimum 3 
years bedside experience, and a minimum qualification of diploma in nursing and incumbent RN license from the country of origin.

R7 "I was recruited by a private agency in India. They gave an announcement through the newspaper regarding the interview. I have contacted them and they told me to take the Prometric exam which I passed with a 50\% mark" (pg.14. Line 460-464).

R9 "I found out about the job opening in an agency in India and I felt I met the criteria so I applied, the agency informed me about taking the Prometric exam. I have to wait based on the vacancies available. I actually waited for at least four years. I applied in Oman also because I want to be with my husband who is working here." (pg. 17. Line 601-607).

\section{Preparation for transition}

Many of the IRN's are unfamiliar with Oman its background in terms of social norms, customs, culture, and tradition. Many did advance personal research, reading, and personally asking former colleagues and relatives that are residents of Oman about the country prior to being deployed. The majority came with little or no understanding about Oman and were surprised about the difference of what is being portrayed in the media about Middle Eastern countries, a Filipino IRN says. Since language is the main barrier to care, many of the IRN improvised ways to learn Arabic prior to being deployed through books, the internet, and by using translation applications.

R2 "I have friends also here in Oman before I came, so I asked them how the people here, the climate the interaction of locals here is to foreign people coming here in the country, mostly my feeling after talking to them I felt relief because they told me that Oman is a good country."( pg.4 line 136-139).

R11 "it was a big transition for me. It was really stressful for me as I needed to endorse my position before leaving the Philippines. The representative from the ministry was very accommodating; it was not that scary. I was given free and comfortable accommodation. I was able to explore some areas near my accommodation. I have this notion before that a Middle East country is very scary. This is because of the news I heard and saw. People have a negative connotation about a Middle Eastern country. Based on the news a Muslim country has more strict regulations compared to my home country. My mental background about a Muslim country is there are a lot of restrictions, especially on social life." (pg.21 line 800-808).

R13 "I did some research regarding Oman, the cultures, the traditions, and especially how to communicate with them. Some basic Arabic language I think I did that, I took some notes, used applications and I researched also about the do's and don'ts when speaking with Omani people because at that time I know that they are very particular, what do you call this, hmm. Like traditional."(pg. 26- line 995-996)

\section{Barriers and challenges}


While many IRN's uses English as a second language to communicate, speaking the native language Arabic was seen as a challenge in the first months of being deployed in Oman. Many felt frustrated about their inability to send the message across patients and felt that it is important that proper training and orientation should have been given by the placement agencies as well as the receiving institutions in Oman. It is apparent that there is no established form of induction and orientation system in place for both the recruiters and the country recipients. When the IRN arrives in Oman they are expected to fill the position readily, sometimes abruptly, and to adjust into the system outright without proper induction and orientation. In many cases, this increases anxiety on the part of the newly hired IRN.

$\mathbf{R} \mathbf{2}$ "In nursing I feel communication is very important when I came here it's also my first time in a foreign country, how I communicated them was using sign language because I didn't know Arabic, sometimes they will laugh because they don't also understand my sign language, and here it's also different simple as saying yes or no other than that I can't understand anything" (pg. 5 line 154-158).

R4 "I didn't know Arabic when I came here too, I'm going to study and all and but slowly I'm learning some patients who know only Arabic so I can reply only yes or no.so what i do is i tell them I don't know Arabic directly they will realize and they will laugh, and I'll just ask somebody to explain to them. (Pg.10 line 320323).

$\boldsymbol{R} 9$ "I had a communication barrier. Until now I have difficulty communicating with a patient because of the language, especially the accent. We have a fear that if he gives wrong communication it will end up in big issues. So we asked help from the locals to translate our communication to the patient. Prefer to be with a local during the shift duty or somebody who can speak Arabic." (Pg. 18. Line 650-653).

R10 "Though the communication is in English sometimes we have encountered miscommunication like the use of words or interchanging of terms. Like English used in an American accent or through British accent example is the use of a torch and a flashlight or the use of lift and an elevator. I think one of my problems is adapting to the language in both Arabic and English. (pg. 21. Line 818-822)

Food is seen as an integral part of culture and tradition. Different countries and nationalities may have different food preferences. For some IRN's adjustment in terms of food was seen as a challenge at first. Varying levels of unfamiliar spices is something new to IRNs coming from the Philippines. Omani food draws from influences of Persian, Arabian, and Indian cuisines which use abundant amounts of spices highly unfamiliar among the Filipino IRN's. Acculturation programs that involve orienting newly hired staff about food, where to get products and supplies that they are familiar with, such as the nearest Asian store and restaurants are important details of the induction and orientation that are not being done when staff from supplying countries reaches Oman.

R2: "Food here, taste different it's a big adjustment so I prepare my food, people are kind but not that sweet like our countrymen they offer and kind, religion-related food restrictions, we are already familiar with Muslim culture because we have also Muslims back home." (Pg.6 line -183-185) 
R14 "The first week, ah, it was, I'm a little bit shocked about the food, because that time, they gave us the food, this biryani because as a Filipino I am not used to that food so at first I did not eat for 2 days. I didn't know where I could go to find or buy products that I could eat at first. (Pg.33 line 1245-1247).

Many IRN says that there were no formal orientation programs or even induction programs in place when they arrived fresh in Oman. Although the staff were cooperative, especially the local nurses were expected to fill the role of the staff on the very first day of their duty without being fully immersed or given a period of adjustment to familiarize themselves with the new environment. A couple of IRN's was promised to be assigned in a special area where they have extensive years of experience as nurses before coming to Oman were later on informed that they will be assigned on other areas than that promised during the interview and contract signing. Some staff were asked to sign a form or document written in Arabic without any explanation of what the contents are which made them really anxious.

R13 "I was really confused because when we were transported from the airport to the accommodation, there was no orientation, there was no one talking to us. This is what we will do and afterward, you will be deployed here, you will temporarily stay here. No, no information at all, in general, just waiting day after day what they will say. For one week we were confused, most of us because we were 20 Filipinos. 10 15 Filipinos in a group when we went here in Oman." I was also promised to be assigned to the ICU, I was later placed in the Health Center (pg. 27- line 1034-1039).

R2 "When I arrived at the guest house, we were informed to report in the Ministry office the following day, we were asked to sign another paper which is written in Arabic, no one explained it to us. I don't speak or read Arabic so I was anxious about it. (166-169).

\section{New work environment experience}

When asked about their new work environment the majority of the IRN's responded that they are generally happy with the health care system in Oman, they were positive also about their Omani co-workers who seem very welcoming and helpful in their period of transition in the workplace. Although a big number of the IRN participants were highly experienced they felt that there is a big difference in the practice of nursing Oman compared to their country of origin. Clinical skills were observed not built on ideal principles as taught in nursing schools.

R8 "I felt welcomed in the first few weeks upon joining my area. The locals and my Omani colleagues are very helpful, but I observed that staff from different nationalities didn't welcome me as much as the locals did. They sometimes don't inform me of what is happening around the area and usually delegate the most difficult patients to me. I felt left behind. (pg. 20 - line 456-459).

R4 "It's still difficult till now because of differing practices depending on where they trained especially. If principles are not followed. I will not mention the nationality, like principles followed are book based some of them do what they are used to. A staff member once asked me to endorse a patient who is not 
assigned to me because she is a female staff member. Conflict with what is taught and what is practiced. (pg. 8 line 255 -262)

Oman and Omanis are known for their welcoming attitude and politeness but for IRN's who are deployed for the first time in the clinical area factors such as language, difference, and practice can lead to miscommunication and can further, escalate into heated arguments among staff and patients or staff to staff. Although rarely experienced some IRN's felt some degree of discrimination from patients and patient relatives. IRN's mostly experiences segregation or discrimination as they feel dissociated from the societal structure and seen only as foreign workers or labor force but not integral to the overall community structure. Discrimination was most felt among differing nationalities rather than with the locals. Dominant nationalities in one area creates an informal community structure that has its own unspoken guidelines and norms in this occasion any other nationalities other than those that are in the majority are seen as foreign or even a threat.

R6 "A patient relative came rushing into me, shouting about his relative, I cannot understand anything, it was only my $2^{\text {nd }}$ week in the unit. I went to my charge nurses. I was really scared. I cried. Most local patients see us only as workers here. (pg. 9. Line 367-369).

R11 "But I have challenges with my direct supervisor. I feel she is biased in terms of her own countrymen. She treats me differently compared to my colleagues from her own country. I find some difficulties because of the language. I cannot understand the way she talks because of the accent. I have difficulty understanding how they articulate words which I find difficult for me to work with them."(Pg.22 line 881883)

While the above findings show very little percentage among IRNs, the majority of the IRN felt welcomed as soon as they joined the clinical area. The following comment is reflective of the many views of the participants.

R14 I feel very blessed working here it because it's the first time for me to work in a foreign country, I felt like I am home because for example my colleagues, they are treating me like family, for example when my mother came here to visit me, they are all like welcoming my mother, they are family like this, and also for the patients when they knew that my mother is coming, they are all excited and happy, that's why I felt more secure, working here in Oman and it is such a nice experience here" (pg.34. Line 1282 -1287)

\section{Variations in practice}

Even upon arriving in Oman the IRN's have a higher expectation of the quality of the healthcare system and service provided in the Sultanate. IRN's coming from low-income countries expect a much better work experience in high-income countries like Oman. The varying practices, as experienced by IRN's, may stem from the diversity of staff working in Oman who handed over some practices to younger staff members with most not done in an ideal way. One IRN appreciates the opportunity to be able to work with sophisticated types of machineries and gadgets that enhance her work experience in Oman. This is 
evidently true among IRN's who reside in low-income countries where there is a scarcity in resources as well as updates in the most recent equipment. There is a high expectation in terms of performance and skills among IRN's recruited since IRN's are seen as specialists with more extensive experience and rigorous academic background.

$R 7$ "It's my first time to see some equipment and machines. Even a simple ventilator looks new to me. The ventilators here are more complicated/complex but more sophisticated and are in the latest model in terms of ventilators. We back home the ventilators we use are the same in all age groups but here in Oman, we use a specific type of ventilator for a specific age group of patients. I am happy and thrilled to use the most updated and sophisticated machines." (pg. 15 line-490-496)

$\mathbf{R} 8$ "before I was working in the neonatal critical care area now I was posted at ward level. I'm used to taking care of neonates now I'm taking care of adult patients. Though the classification of patients changed from critical care to the independent type of patient the number of the patient increased. I think the workload is heavier this time. If given the chance I would like to go back to my specialization which is the neonatal intensive care unit. I was only posted because of the vacancies in the ward. Initially, I was surprised and had difficulties being able to sign the ward level but my manager helped me to adjust. I feel tired sometimes but there is teamwork. Some days are busy, some days are hectic. But we try to manage our work." (pg. 16. Line 564-572)

Gender segregation is apparent in Oman similar to other Middle Eastern countries that practice Islam as a religion. Male IRN's are expected to abide by the norms and traditions related to handling female patients and the customs traditionally followed while interacting with them. IRN's coming from India and the Philippines are trained to handle patients across gender and age and are expected to perform tasks equivalent to those of female nurses.

R15 "Me as a male staff, I cannot just do the nursing interventions or any procedures ordered by the doctor to perform on female patients, we always give considerations that if there's like physical contact with the patient, it's already expected that a female staff will perform it on a female patient, like if the patient has permanent access which is located in the subclavian, so male staff like me doesn't do it, so we already allow only the female staff to perform that.". (pg.39 line 1471-1475).

\section{Adjustment and acculturation}

Participant IRN's expressed the adjustment period and the cultural experiences they went through, most adjusted easily owing it to their own cultural background that allows acceptance and flexibility, supportive staff members, and the availability of immediate family and relatives have greatly impacted their smooth and steady transition in the Omani society. For some adjustments were difficult as it is their first job abroad, while those who have already worked in other countries especially countries in the Middle East adjustment was faster. 
R2 "I think now I'm more confident with communicating although I still have difficulty with the Arabic language but at least now somehow I can communicate, I can ask simple questions and I can understand their answers also. The practice is mostly similar back home nursing procedure is almost the same. (pg.6 line 178-181)

R2 "I think from my experience as a Filipino nurse are trained to adjust to different environments, we are trained even in the Philippines we are trained to be resourceful, like when we don't have ideal materials and resources we are able to give the interventions to our patient that helped me a lot when I came here in Oman." (pg. 6 line 178-181).

R8 "Being with my husband or my family helps me in adopting in Oman. I feel I can express being free or more freedom here in Oman compared to my previous work. I feel relaxed. We can go out, we can go to the beach and we can enjoy it. We can also practice our religion by going to the church, going to the park. In terms of lifestyle like wearing clothes, Oman and Indian have similarities. Very supportive in charge or manager and helpful preceptors which guide us and correct us in the ward." (Pg. 15 line 575-580).

$\boldsymbol{R} \mathbf{9}$ "My preceptor is one of the keys for me to adopt in my professional work. There is not much restriction or someone compels us to do something that makes us comfortable staying in Oman. If we are comfortable we will adapt easily. Maybe I am with my family, that's why I adapt easily. I don't have a problem with my social lifestyle here in Oman." (Pg.18 line 661-665)

R15 "Professionally, my adjustment was I guess not that easy since it is my first time to work abroad especially in Arab country where the culture is like different from ours, but I was able to adapt more than I expected because I think here, a month after, more Filipino staff came so I had some resemblance, I mean, my adjustment became easier that time since I have more friends, more Filipino friends here in Oman" (pg.40 line 1522-1526).

\section{Improving the hiring process}

Participants expressed many unmet expectations upon joining Oman in terms of the hiring system and processing, living conditions, work arrangements that were immediately resolved or managed. The biggest challenge among the IRN's upon arrival is the lack of proper training, orientation, and induction programs to ease the transition into the new work environment. Almost all participants mentioned the lack of an acculturation program that will help them assimilate into a country totally foreign to them. It is known that language can be a major obstacle in the practice of nursing since caring is largely dependent on effective communication with the patient, relatives and coworkers. The lack of basic Arabic courses and culture integration programs from both country origin placement agencies and catchment institutions in Oman is seen as a factor in the difficulty transition experience of IRN's especially those who are working abroad for the first time. The high cost of hiring on both the IRN's who pays placement fees and the receiving catchment institution in Oman that shoulders the recruitment process demands a more systematic approach in transitioning Internationally recruited nurses from non -Arabic speaking 
countries. IRN's are left to fend for themselves to immerse and learn while taking on the responsibility in a new environment that can at times become too overwhelming.

$\boldsymbol{R} 11$ "the agency notified me that within a month I need to go to Oman or else my visa will expire. From my work, I need to notify the hospital two months prior to my resignation. It was a dilemma for me whether to resign or not. It will take hours for me to travel from my home to the recruitment agency. The reason for me going there personally is because no one is picking up the phone. I need to go there just to ask or inquire about the question. It took a lot of effort for the processing of my papers. I don't have any idea about Oman. It was an abrupt notification from an agency, a great distance between the agency in my home and a long waiting time for the processing of my papers."(pg.21 line 783-790).

R2 "The agency should provide training specially Arabic language, my first struggle is language, maybe they should include some small curriculum about the language although the agency gave some list of words like greetings but being a nurse communication is very important to be included before they are deployed.(pg.6 line 188-190).

R9. "I feel the recruitment agencies are profiting money from the applicants. I wish that they would orient us with the culture of the country, especially on how to communicate. I hope they can teach basic Arabic language. At least two sessions for Arabic class." I hope recruitment agencies will move from private to government. (pg. 18- line 668-670).

$\mathbf{R} 10$ "I hope the agency will provide an orientation program regarding Oman. Particularly if they can provide at least important Arabic words for the nurses to learn before going to Oman. If the agency can provide details on the posting details or area where we will be working on. This makes us confuse and makes us worry" (pg.20 line 755-758)

R14 "ah I think they should explain more to the nurses before, especially the culture in which they will be going so when the nurses go there if they will not be in shock, I used to cry because it's hard working in a new environment there's so much anxiety" (pg. 35 line 1326-1327).

\section{Impermanence and Omanization}

Participants were all very thankful for the opportunity given to them when they were asked to work as nurses in Oman. The promise of better income, and working conditions were motivating factors identified by the participants but in spite of these comforts provided in the country the looming perspective of termination, job loss, and replacement are felt. The nationalization drive may put an end to the dreams of IRN's in Oman. Instability in the economic standing, clamor for better job positions for locals and the pandemic situation has escalated the move to replace IRN's with locally trained nurses.

R9 "I am very happy with work here in Oman, but there's also fear and anxiety. We are not permanent here, every year more and more nurses are being terminated because of Omanization. We have to be prepared" (Pg. 19. line 654-656) 


\section{Discussion}

The study researchers planned to meet the ideal variation in the sample. Variation in terms of the geographical assignment was met. Data saturation was attained as observed with the repeated and similar views and very few unexpected themes. The general findings of the study are consistent with the other similar studies about foreign-trained nurses in developed countries (Jose, 2011, Blythe \& Baumann, 2009. \& Higginbottom 2011). Overall the study revealed that the recruited participants consisted of well trained and experienced nurses from the Philippines and India. The Philippines is known as the largest supplier of foreign-trained nurses in the world (Masselink \& Daniel Lee,2012). Most of the participant IRN's are holders of Bachelor's degree and a diploma in nursing and have some prior experience working in other countries before joining Oman. IRNs educational qualifications and experiences are valued this is contrary to other developed countries who undervalue the training and educational qualifications of foreign-trained nurses (Eriksson, Berg, \& Engström. 2018). The study reveals that the IRN's main motivation in working abroad is to secure better income and experience better working conditions (Montayre, Montayre, \& Holroyd, 2018). The nurses choice for the migration country is both random and based from opportunity (Nortvedt et.al 2020). The hiring of foreign-trained nurses in Oman is mediated by parties such as recruitment agencies to local governmental catchment institutions as a part of the hiring process, in Oman although the scheme has been practiced for years and is seen as effective on the point of view of the receiving country, it takes a lot of challenge for the applicants to be able to land a job without being exploited. An existing ethical recruitment system is not seen in place or if it exists is not implemented (Shaffer, Bakhshi \& Jacobs, 2018). The study shows that the absence of an ethical recruitment system to both parties may eventually lead to more difficult adjustment and the transition period for the newly hired foreign-trained nurses reporting insufficient orientation, discriminatory practices such that of wages and benefits as well as unit assignments (Pittman, Davis \& Shaffer et.al C., 2014). The transition period for IRN's has posed challenges and barriers to enable them to smoothly adapt to their new environment. Studies show that it is imminent that foreign-trained nurses experience differences in nursing practice and cultural values, communication problems, discrimination, and competency recognition if an integration program is not in place. (Primeau, Champagne \& LavoieTremblay 2014, Cooper et al 2007). The adjustment process involves being aware of both the cultural and religious background of the country placement the IRN's are deployed. Oman a country in the Gulf region is a Muslim country rich in culture and tradition homogenous to UAE and KSA study was conducted to assess the cultural and religious needs of overseas nurses in these countries it revealed and identified three important education needs for overseas nurses that are related to Islamic principles, kinship and social norms as well as basic Arabic communication skills (Al-Yateem, AlYateem \& Rossiter, 2015). Most IRN's experienced difficulty with language that serves as a barrier to care and communication, this seems to hinder the competency of the nurses since communication is an important aspect of the daily care for the patient and establishing communication and relationship with colleagues as well as the patient's relatives. (Alosaimi \& Ahmad, 2016). IRN's agree that a certain level of language proficiency is required when working at the bedside and see that it's imperative for the recruitment agencies to at least provide the nurses for deployment to undergo training and orientation on basics in language, culture, and religion 
(Pittman, Davis, \& Shaffer et.al, 2014). Although most IRN's have previously been employed in other countries prior to working in Oman they see their new work environment as a way to further improve themselves in terms of gaining more training and knowledge about their specialties and as well as learning to manipulate new equipment which is seldom used in their country of origin. IRN sees employment in first world countries such as Oman as a part of a two-part migration process to smoothly transition into other western countries such as the UK, USA, Canada, New Zealand, and Australia (Ennis \& Walton-Roberts, 2018). There is an observed variation in nursing practice that stems from a highly internationalized healthcare workforce. Each individual IRN's carries with them their practice, knowledge, and experience that greatly contribute to the evolution of the practice and care in Oman. Although seen as a positive point, differing practices may cause confusion and delayed adaptation among IRN's who are newly transitioning to the system. The levels and skills of IRN's from developing countries are greatly influenced by their previous work experience and practice background. (Bruyneel, Li \& Aiken et al, 2012). There is an intensified implementation of the workforce nationalization in the previous years, the government plans to implement Omanization to create jobs for local graduates up to $70 \%$ of government posts for nurses are expected to be filled in the next few years. This has increased migrant nurses' vulnerability. The majority of the respondents aired their apprehensions and anxiety about the possibility of them losing their jobs. There is a trend of declining Omanization from 2010-2015 may evidently be seen in the coming years. The need for experienced well-trained expatriate nurses within the Omani healthcare system especially in special areas remains (Ennis \& Walton-Roberts, 2018 ). The prospects of $100 \%$ Omanization is still far from being realized due to the barriers and challenges faced by local nurses such that of work-life balance, preparation of nurses, and the pacing of replacements (Al-Riyami , Fisher \& Lopez , 2015).

The present study found that IRN's greatest concerns are related to assimilating the culture, practices, and language. The lack of proper integration programs from the recruitment agencies and in catchment institutions in Oman makes the adjustment period challenging. Language seen as the biggest obstacle among IRNs has resulted in miscommunications, workplace displacements, microaggressions, and discriminations from both patients, relatives, and co foreign and local workers. The hiring and recruitment process needs to be revisited so as to avoid exploitation and undue treatment from the agencies hiring in their respective origin countries.

There is a limitation to this study. Qualitative studies are subjective analysis and interpretations are highly based on the participant's experiences. Participants may have provided information that is socially desirable or acceptable. Lastly, the study was conducted in the midst of COVID-19 pandemic wherein the health protocols limited the data collection to recorded phone conferences and interviews which was originally planned to be given face to face, the lack of physical interaction and observation may have fully conveyed the information shared by the participants.

Implication for nursing practice 
The outcomes of the study generated important information and outlook on the lived experiences of foreign-trained nurses in Oman. The understanding of the nurse's lived experiences provides insight on what are the unexplored issues surrounding recruitment, transition, and the nurse's adaptation to Oman. The study results provide a greater understanding of the barriers and challenges faced by IRN's in Oman and also revealed the good practices and environments enjoyed by the IRN's during their stay in the country. A systematic and ethical recruitment system is required in order to resolve issues related to integration, orientation, and assimilation in the catchment institutions. Basic orientation programs should be in place to acculturate the foreign-trained nurses not only about the work environment but also about the social, religious, and cultural environment they are new with. Furthermore, the study dealt with relevant concerns related to workforce nationalization. Lastly, the study results can be used for the future development of acculturation programs to fully integrate IRN's deployed to Oman.

\section{Conclusion}

This phenomenological study explored the experience of internationally recruited nurses in Oman. The themes derived from the study are significant to the experiences felt by the IRNs. IRN sees Oman is a country that provides opportunities for better wages, professional development, and a highly tolerant society that respects social and personal differences. The study results showed the need for a more systematic and ethical recruitment strategy that enhances IRN's full integration to the host country's social, religious, and cultural system. It was also found that language is an integral part of the IRN's assimilation therefore the need for a formal and structured training and orientation program must be in place in both recruiting agencies and the catchment facilities in Oman. Even with the continuous effort to nationalize the health workforce, qualified and well-experienced IRN's are still seen as vital contributors to the overall progress and development of the Oman healthcare system.

\section{Declarations}

Funding

No funding received for this study

Conflict of Interest

The authors declare no conflict of interest

\section{References}

Al Maqbali, M. R., Al Omari, O., Slimane, S., \& Balushi, N. A. (2019). The Nursing Profession in Oman: An Overview. Nursing science quarterly, 32(4), 322-325. https://doi.org/10.1177/0894318419864346

Ali, P. A., \& Watson, R. (2018). Language barriers and their impact on provision of care to patients with limited English proficiency: Nurses' perspectives. Journal of clinical nursing, 27(5-6), e1152-e1160. 
Alosaimi, D. N., \& Ahmad, M. M. (2016). The Challenges of Cultural Competency Among Expatriate Nurses Working in Kingdom of Saudi Arabia. Research and theory for nursing practice, 30(4), 302-319. https://doi.org/10.1891/1541-6577.30.4.302

Al-Riyami, M., Fischer, I., \& Lopez, V. (2015). Nurses' perceptions of the challenges related to the Omanization policy. International nursing review, 62(4), 462-469. https://doi.org/10.1111/inr.12221

Al-Yateem, N., AlYateem, S., \& Rossiter, R. (2015). Cultural and Religious Educational Needs of Overseas Nurses Working in the Kingdom of Saudi Arabia and the United Arab Emirates. Holistic nursing practice, 29(4), 205-215. https://doi.org/10.1097/HNP.0000000000000095

AlYateem, S. \& Al-Yateem, N. (2014). The Experience of Overseas Nurses Caring for Muslim Patients in Kingdom Of Saudi Arabia and Uae: A Qualitative Study. International Journal of Research in Nursing, 5(1), 17-26. https://doi.org/10.3844/ijrnsp.2014.17.26

Blythe, J., \& Baumann, A. (2009). Internationally educated nurses: profiling workforce diversity. International nursing review, 56(2), 191-197. https://doi.org/10.1111/j.1466-7657.2008.00699.x

Bond, J., \& Bond, S. (1994). Sociology \& Healthcare -an introduction for nurses and healthcare professionals . Edinburgh: Churchill Livingstone .

Bruyneel, L., Li, B., Aiken, L., Lesaffre, E., Van den Heede, K., \& Sermeus, W. (2013). A multi-country perspective on nurses' tasks below their skill level: reports from domestically trained nurses and foreign trained nurses from developing countries. International journal of nursing studies, 50(2), 202-209. https://doi.org/10.1016/j.jinurstu.2012.06.013

Chenowethm, L., Jeon, Y. H., Goff, M., \& Burke, C. (2006). Cultural competency and nursing care: an Australian perspective. International nursing review, 53(1), 34-40. https://doi.org/10.1111/j.14667657.2006.00441.x

Colaizzi, P. (1978). Psychological research as a phenomenologist view. In R. Valle , \& M. King , Existential Phenomenological Alternatives to Psychology (pp. 48-71). New York : Oxford University Press.

Cooper, M., Grywalski, M., Lamp, J., Newhouse, L., \& Studlien, R. (2007). Enhancing cultural competence: a model for nurses. Nursing for women's health, 11(2), 148-159. https://doi.org/10.1111/j.1751486X.2007.00130.x

Daniel, P., Chamberlain, A., \& Gordon, F. (2001). Expectations and experiences of newly recruited Filipino nurses. British journal of nursing (Mark Allen Publishing), 10(4), 254-265. https://doi.org/10.12968/bjon.2001.10.4.5374 
Drennan, V. M., \& Ross, F. (2019). Global nurse shortages-the facts, the impact and action for change. British medical bulletin, 130(1), 25-37. https://doi.org/10.1093/bmb/ldz014

Ennis, C. A., \& Walton-Roberts, M. (2018). Labour market regulation as global social policy: The case of nursing labour markets in Oman. Global social policy, 18(2), 169-188.

https://doi.org/10.1177/1468018117737990

Eriksson, E., Berg, S., \& Engström, M. (2018). Internationally educated nurses' and medical graduates' experiences of getting a license and practicing in Sweden - a qualitative interview study. BMC medical education, 18(1), 296. https://doi.org/10.1186/s12909-018-1399-4

Higginbottom G. M. (2011). The transitioning experiences of internationally-educated nurses into a Canadian health care system: A focused ethnography. BMC nursing, 10, 14.

https://doi.org/10.1186/1472-6955-10-14

Hill , A., \& Chen, L. (1996). Omans Leap to a Good Health: A Summary of Rapid Health Transition in the Sultanate of Oman . World Health Organization Children's Fund .

Index Mundi: Oman Demographic Profile 2014. (n.d.). Retrieved June 12, 2019, from http://www.indexmundi.com/oman/demographics_profile_html.

Jose M. M. (2011). Lived experiences of internationally educated nurses in hospitals in the United States of America. International nursing review, 58(1), 123-129. https://doi.org/10.1111/j.14667657.2010.00838.x

Masselink, L. E., \& Daniel Lee, S. Y. (2013). Government officials' representation of nurses and migration in the Philippines. Health policy and planning, 28(1), 90-99. https://doi.org/10.1093/heapol/czs028

Matiti, M. R. \& Taylor, D. (2005). The cultural lived experience of internationally recruited nurses: a phenomenological study. Diversity in Health and Social Care, 2(1), 7-15.

MOH. (2017). Annual Health Reports: Chapter 4 Human Resources for Health . Muscat : Ministry of Health .

Montayre, J., Montayre, J., \& Holroyd, E. (2018). The global Filipino nurse: An integrative review of Filipino nurses' work experiences. Journal of nursing management, 26(4), 338-347.

https://doi.org/10.1111/jonm.12552

Nortvedt, L., Lohne, V., \& Dahl, K. (2020). A courageous journey: Experiences of migrant Philippine nurses in Norway. Journal of clinical nursing, 29(3-4), 468-479. https://doi.org/10.1111/jocn.15107

Nurse, M. (2013, March 30). Minority Nurse: Professional Nursing in Oman. Retrieved from https://minoritynurse.com/?s=professional+nursing+in+oman. 
Patel-Kerai, G., Harcourt, D., Rumsey, N., \& Naqvi, H. (2010). The Cultural lived experience of internationally recruited nurses: a phenomenological study. Diversity \& Equality in Healthcare.

Pittman, P., Davis, C., Shaffer, F., Herrera, C. N., \& Bennett, C. (2014). Perceptions of employment-based discrimination among newly arrived foreign-educated nurses. The American journal of nursing, 114(1), 26-36. https://doi.org/10.1097/01.NAJ.0000441792.93279.29

Polit, D., \& Tatano Beck, C. (2014). Essential of Nursing Research (8 ed.). Philadelphia: Wolter Kluwer Health.

Primeau, M. D., Champagne, F., \& Lavoie-Tremblay, M. (2014). Foreign-trained nurses' experiences and socio professional integration best practices: an integrative literature review. The health care manager, 33(3), 245-253. https://doi.org/10.1097/HCM.0000000000000018

Sandelowski, M., Docherty, S., \& Emden, C. (1997). Focus on qualitative methods. Qualitative metasynthesis: issues and techniques. Research in nursing \& health, 20(4), 365-371.

https://doi.org/10.1002/(sici)1098-240x(199708)20:4<365::aid-nur9>3.0.co;2-e

Shaffer, F., Bakhshi, M., \& Jacobs, A. (2018). Advocating to Protect Our Nurses: Addressing Unethical Recruitment of Foreign-Educated Nurses. Nursing administration quarterly, 42(2), 107-114. https://doi.org/10.1097/NAQ.0000000000000274

Valdez, GFD. (2016). The Filipino Nurses in the Diaspora: Contextualizing Workforce Diversity \& Cultural Adaptability: A Middle Eastern Perspective. Journal of Nursing Care : 6th World Nursing and Healthcare Conference. London: OMICS.

\section{Table}

Table 1 is available in the Supplementary Files

\section{Supplementary Files}

This is a list of supplementary files associated with this preprint. Click to download.

- Table1RerspondentsProfile.docx 